\title{
A Wake Interaction Model for the Coordinated Control of Wind Farms
}

\author{
Lin Pan, Holger Voos, Yumei Li \\ Interdisciplinary Centre for \\ Security, Reliability and Trust, \\ University of Luxembourg, Luxembourg \\ Email: lin.pan@uni.lu, \\ Holger.Voos@uni.lu,yumei.li@uni.lu \\ Yuhua Xu \\ School of Finance, \\ Nanjing Audit University, \\ Jiangsu 211815, China \\ Email: yuhuaxu2004@163.com
}

\author{
Mohamed Darouach \\ Research Center for \\ Automatic Control of Nancy \\ (CRAN UMR, 7039, CNRS), \\ University of Lorraine, France \\ Email: mohamed.darouach@univ-lorraine.fr \\ Lin Pan, Shujun $\mathrm{Hu}$ \\ School of Electric \& Electronic Engineering, \\ Wuhan Polytechnic University, \\ Wuhan, China \\ Email: hushj@whpu.edu.cn
}

\begin{abstract}
In all the processes of Wind Energy (WE) utilization, the Wind Power (WP) assessment is critical stage for all the Wind Farms (WFs). This paper is focused on the WE systems in Luxembourg. It describes the overview of the wind resources in all the WFs and presents an Unified Cooperation Wake Model (UCWM) and Coordination and Optimization Control (CnOC) for WFs. Based on WP assessment of WFs, the statistical method is used to model the distribution of wind speed and Wind Direction (WD). Some simulation figures about the wind rose and Weibull distribution demonstrate the detailed description and assessment of WP. These assessments are expected to enhance the effectiveness of WP exploitation and utilization in WFs of Luxembourg.
\end{abstract}

\section{INTRODUCTION}

It is well known that WE is widely recognized to be one of the most cost-efficient renewable sources of energy. With the increase of global wind-generation capacity in the last five years, WE has also become the fastest-growing electrical energy in the world. In order to enhance the utilization efficiency of WE, the most efficient way is to utilize existing WFs through improving control techniques and algorithms. At present, WE systems are being inclined to develop into large-scale distributed and coordination systems where there are even more than eighty individual Wind Turbines (WTs) in operation. In contrast to the conventional power plants, e.g. nuclear power, thermal power, hydropower, etc. [1], [2], [3], [4], [5], [6], [7], these wind devices and equipment are expected to operate and provide high quality power (Such as: Safe, Stable, Controllable and Predictable (SSCP)) at the lowest possible cost.

In recent years, the research and development of WE harvesting systems were focused on optimizing different aspects of the WT in order to improve its Cost of Energy (CoE). Increase performance of the control system by optimizing the WT controller [1], [2] is one of the most important ways to enhance CoE of WT. WTs are often located in so called wind

978-1-4673-7929-8/15/\$31.00 @ 2015 IEEE parks or wind farms together with other turbines as so to reduce costs by taking advantage of economies of scale. Turbines in WFs can be located along a single line, in multiple lines, in grids, in clusters or in configurations based on geographical features, prevailing WD, access requirements, environmental effects, safety, prior and future land use including ranch-land and farmland, and visual impact [3], [4].

Generally, the research on control of an array of WTs in WFs is more complex than control of single-WT setting because of the aerodynamic interactions among the array WTs. Therefore, the control on WFs is a more challenging research. In contrast to use single-WT control algorithms only, optimizing WP capture in WFs by coordination and optimization control of WFs will no doubt increase the utilization efficiency of WE. The potential for improving performance and function, increasing WP capture as well as optimizing electricity loads among the WFs, have led to novel research efforts in coordination and optimization control of WFs. One method for dealing with these aerodynamic interactions is to develop and use wake models in the distributed and optimization control algorithms. An alternative method is to develop an online control approach where each WT adjusts its own induction model coefficients in response to the information of local WFs, such as the WP generated by individual WT, local wind conditions, local wind speed, local WD, local density of air, or interacted information regarding neighbor WTs. Here, the goals are to develop coordination and optimization control approaches that permit the field of WTs to reach a desirable set of model coefficients, which will lead to better system level behavior, for example, WP maximization or electricity loads minimization, without the need for complex modeling of the WFs [5], [6].

In view of Luxembourg locating in the western central area of European, and there are abundant wind resources to tap into Luxembourg. There are currently more than 16 WFs established in the different places of Luxembourg. The list of WFs in Luxembourg is shown in the table I [8]. As an example, 
TABLE I. LIST OF WIND FARMS (WFS) IN LUXEMBOURG[8].

\begin{tabular}{|c|c|c|c|}
\hline Country/City & Name & Number of turbines & Power \\
\hline Luxembourg/Binsfeld & Binsfeld & 5 turbines & $11,500 \mathrm{~kW}$ \\
\hline Luxembourg/Boxhorn & Boxhorn & 1 turbine & $800 \mathrm{~kW}$ \\
\hline Luxembourg/Brachtenbach & Brachtenbach & 1 turbine & $600 \mathrm{~kW}$ \\
\hline Luxembourg/Derenbach & Derenbach & 3 turbines & $1,800 \mathrm{~kW}$ \\
\hline Luxembourg/Doennange & Doennange & 1 turbine & $800 \mathrm{~kW}$ \\
\hline Luxembourg/Reimberg & Reimberg & 2 turbines & $1,200 \mathrm{~kW}$ \\
\hline Luxembourg/Remerschen & Remerschen & 1 turbine & $600 \mathrm{~kW}$ \\
\hline Luxembourg/Wand a Waasser & Wand a Waasser & 3 turbines & $1,500 \mathrm{~kW}$ \\
\hline Luxembourg/Wandpark Haardwand & Wandpark Haardwand & 4 turbines & $2,400 \mathrm{~kW}$ \\
\hline Luxembourg/Mompach & Burer Bierg & 4 turbines & $8,000 \mathrm{~kW}$ \\
\hline Luxembourg/Flebour & Flebour & 3 turbines & $7,050 \mathrm{~kW}$ \\
\hline Luxembourg/Kehmen-Heischent & Kehmen-Heischent & 7 turbines & $12,600 \mathrm{~kW}$ \\
\hline Luxembourg/Mompach & Pafebierg & 4 turbines & $2,000 \mathrm{~kW}$ \\
\hline Luxembourg/Heinerscheid(Part 1) & Wandpark Hengischt S.A./Gemeng Hengischt & 3 turbines & $1,800 \mathrm{~kW}$ \\
\hline Luxembourg/Heinerscheid(Part 2) & Wandpark Hengischt S.A./Gemeng Hengischt & 5 turbines & $5,000 \mathrm{~kW}$ \\
\hline Luxembourg/Heinerscheid(Part 3) & Wandpark Hengischt S.A./Gemeng Hengischt & 3 turbines & $5,400 \mathrm{~kW}$ \\
\hline Luxembourg/Weiswampach & Weiswampach & 1 turbine & $2300 \mathrm{~kW}$ \\
\hline
\end{tabular}

Fig. 1 shows Kehmen-Heischent WF, which is one of the large WFs in Luxembourg [9].

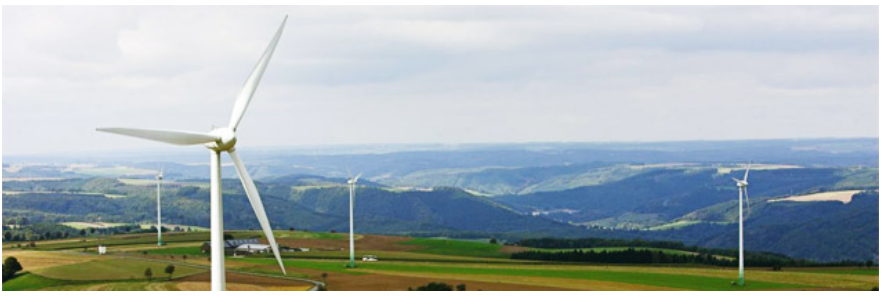

Fig. 1. The portraits of WF Kehmen-Heischent in Luxembourg [9].

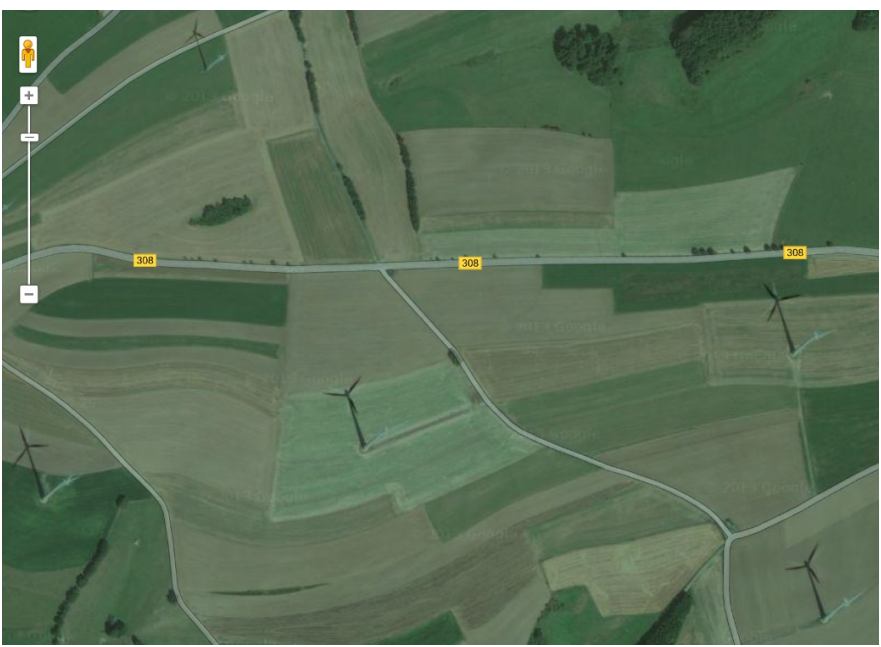

Fig. 2. The portraits of Kehmen-Heischent wind farm (Luxembourg)[10].

Fig. 2 gives the real map of Kehmen-Heischent WF in Luxembourg [9]. From a control perspective, the complexity of large scale farms is handled by a hierarchical approach, separating the control into control on the operator level, the WF level and control on the single WT level [7]. The operator level controller serves the demands from the network operator that gives a set point for active and reactive power for the whole farm combined with one of several operational modes, e.g. maximum energy production, rate limiting, balancing, frequency control, voltages control, or delta control. On the WF level, the set points for the overall WF are decomposed into set points for the single WT, taking the various interdependencies into account. On the lowest control level, the single WT level, the respective set points are achieved via control approaches as described in [7]. Therefore, the most challenging task is the farm level control. Moreover, inspired by the above works and discussions, we agree that the key and core research contents are to operate WFs with the efficient methods, to improve WP, power quality, and energy capture of the overall WTs. At the same time, for reducing complex structural electricity loading, advanced and hybrid control measures have to be applied on the WFs. However, so far, there is still lack of effective control method of WFs which requires further research and development. All factors mentioned above motivate our research in the field.

The main problem in WFs is the fact that the pure control on the single-WT is not completely sufficient because of complex interactions among the WTs. First of all, all WTs in a farm are connected to the same Electrical Grid (EG) and each individual WT therefore has an influence on the quality or output of the generated electrical power. In addition, there are also aerodynamic interdependencies among the WTs because each WT influences the airstream that flows through the WF. Also these flows influence the wind conditions and hereby the power generation as well as the electricity structural load on other WTs. Furthermore, aerodynamic and electrical interaction among WTs in a WF also result in the losses of energy-capture, which have to be recovered by a suitable control measures. Therefore, the overall control strategy of WFs is currently a very important research topic and also the main objective which goes far beyond the control method of a single WT.

The paper is organized as follows: In section II, a class of WT power capture and power model description are introduced. In section III, an Unified Cooperation Wake Model (UCWM) for WF are obtained and discussed. The wind rose and wind Weibull probability density distribution are presented in section IV. Finally, the conclusion is summarized in section V. 


\section{WT POWER CAPTURE AND POWER MODEL DESCRIPTION}

In this section, we introduce the control parameters of a WT by its induction coefficients which represent the fractional decrease in wind speed between the free stream conditions and those seen at the rotor plane. For the optimization of a WF, it is too complex to model all the states of the WTs. The wind model can been parameterize by the induction factors as opposed to more traditional control parameters, for example, tip-speed ratio and pitch angle, to provide a more compact representation of the WF model. More specifically, the power generated by WT- $i$ is characterized by the following equation [2].

$$
P_{i}\left(a_{i}, v_{i}\right)=\frac{1}{2} \rho \pi R^{2} v_{i}^{3} \eta C_{P}\left(a_{i}\right)\left(\lambda_{r i}, \beta_{r i}\right)
$$

where $\rho$ is the density of air, $R$ is the radius of WT, $\pi R^{2}$ is the area swept by the turbine blades, and $v_{i}$ is the average "inlet" wind speed for turbine $i$. The $C_{P}\left(a_{i}\right)$ is the power efficiency coefficient which takes on the form:

$$
C_{P}\left(a_{i}\right)=4 a_{i}\left(1-a_{i}\right)^{2} .
$$

In the equation (1), as a function of the reference values for the Tip Speed Ratio (TSR) $\lambda_{r i}$ and the pitch angle $\beta_{r i}$ with $\eta=\eta_{d} \eta_{g}$ representing the overall turbine efficiency:

$$
\lambda_{r i}=\frac{\omega_{r i} R}{V_{i}}
$$

$\omega_{r i}$ is the speed of the WT. The total power generated in the WF is simply:

$$
P_{\text {total }}(a)=\sum_{i \in N} P_{i}(a)
$$

In order to obtain a sufficiently large correlation coefficient $\lambda_{r i}$, we choose the mean density of air is $1.250 \mathrm{~kg} / \mathrm{m}^{3}$ in Luxembourg 2014, and the radius of WT as $66 \mathrm{~m}$ for WF of Kehmen-Heischent in Luxembourg, and select the following mathematical formula [11]:

$$
\begin{aligned}
C_{p}\left(\lambda_{i}, \beta_{i}\right)= & -0.2000+0.2000 \lambda_{i}-0.007000 \beta_{i} \\
& -0.020000 \lambda_{i}^{2}+0.003000 \lambda_{i} \beta_{i}+0.000400 \beta_{i}^{2} \\
& +0.000700 \lambda_{i}^{3}-0.000200 \lambda_{i}^{2} \beta_{i}-0.000100 \lambda_{i} \beta_{i}^{2} \\
& +0.000001 \beta_{i}^{3}, \quad i \in\{1,2, \cdots\} .
\end{aligned}
$$

From the Fig. 3, we can see that function Power coefficient $C_{p}$ is very well fit with the variables: $\lambda$ and $\beta$.

The dynamic representation of the WT in a switched system has been investigated by authors in [4]. The dynamic model of the integrated system can be represented as:

$$
\dot{\omega}=\frac{1}{J_{r}}\left(\frac{\pi}{8} D_{r}^{2} \rho_{a i r} C_{p} \frac{v_{\omega}^{3}}{\omega}-\tau G_{r}\right)
$$

where $D_{r}$ is the turbine rotor diameter, $\rho_{\text {air }}$ is the density of air, $v_{\omega}$ is the input wind speed, $\omega$ is the turbine rotor speed, $J$ is the combined rotational inertia of the rotor, gearbox, generator, and shafts, $G_{r}$ is the gearbox gear ratio defined as the generator shaft speed over the rotor shaft speed, $\tau$ is the generator torque and $C_{p}$ is the power coefficient that measures how effectively

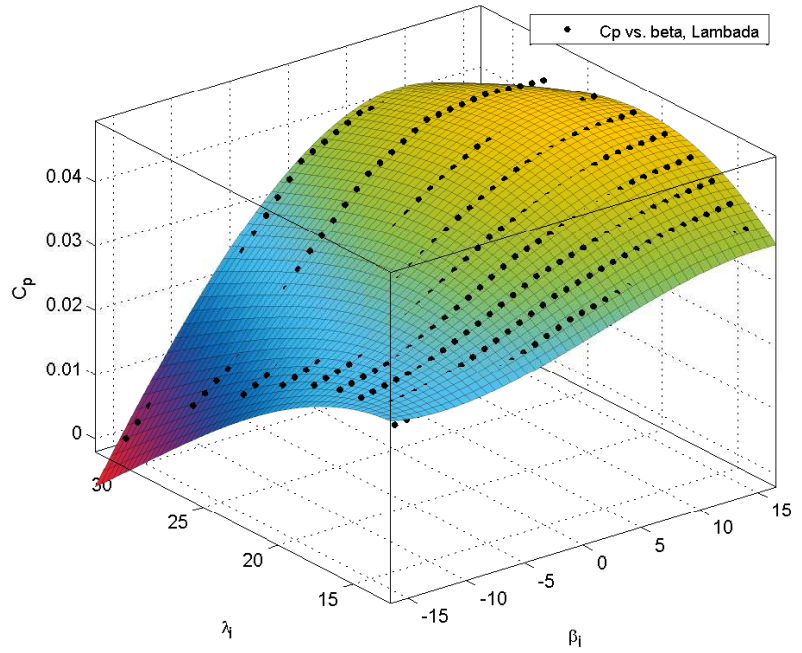

Fig. 3. The portraits of surfaces for power coefficient $C_{p}$ with TSR $\lambda_{i}$, and pitch angle $\beta_{i}$.

the WE is being converted to mechanical energy. It is a nonlinear function of the blade pitch angle $\beta$ and the tip speed ratio $\lambda$ :

$$
C_{p}=f(\lambda, \beta)
$$

The tip speed ratio can be expressed as:

$$
\lambda=\frac{\omega D_{r}}{2 V_{\omega}} \text {. }
$$

We locate 12 WTs as the position matrix in a WF with the software SimWindFarm [12]:

$$
\begin{aligned}
& {\left[\left(x_{1}, y_{1}\right) ;\left(x_{2}, y_{2}\right) ; \ldots ;\left(x_{12}, y_{12}\right)\right] } \\
= & {[(0,100) ;(400,1000) ;(800,100) ;(1200,100) ;} \\
& (0,500) ;(400,500) ;(800,500) ;(1200,500) ; \\
& (0,900) ;(400,900) ;(800,900) ;(1200,900)] .
\end{aligned}
$$

The local mean wind speed $(\mathrm{m} / \mathrm{s})$ is $3.2042 \mathrm{~m} / \mathrm{s}$, the WTs' intensity is 0.1 , WF's length $(\mathrm{m})$ is $1300 \mathrm{~m}$. WF's width $(\mathrm{m})$ is $1100 \mathrm{~m}$, and Grid size $(\mathrm{m})$ is $15 \mathrm{~m}$. The selected simulation time (s) is 1000s. By using the SimWindFarm Toolbox [12], we get the following the portrait of 12 WTs in a WF of Luxembourg as shown in the Fig. 4.

Consider a one dimensional array of $N$ identical WTs as shown in Fig. 5. Turbine $T_{i j}$ is located downstream of turbine $T_{1 j}$ by a distance $x_{i}$. The air flow velocity upstream of turbine $T_{1 j}$ is assumed to be uniform with speed defined as $v_{\infty}$. It is further assumed that all turbines are perfectly aligned with the direction of the upstream airflow, such that the air flow is orthogonal to each turbines plane of rotation. The power generated by the WF depends on the free-stream wind speed and the control actions of each turbine.

The mutual aerodynamic interaction of turbines in a WF is not as well understood as the electrical interconnection of the turbines. While WFs help to reduce the average cost of energy compared to widely dispersed turbines due to economies of scale, aerodynamic interaction among WTs can decrease the 


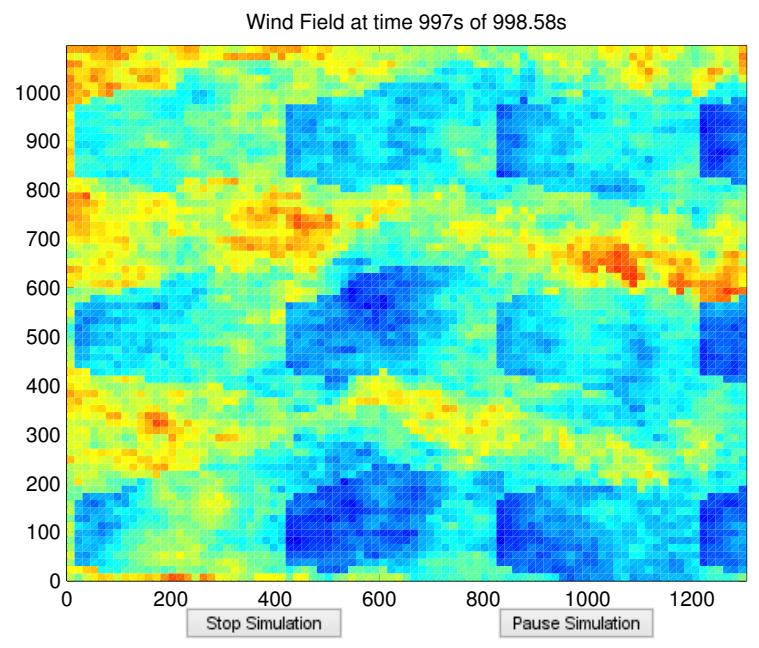

Fig. 4. The portrait of 12 WTs in a WF of Luxembourg.

total energy converted to electricity compared to the same number of isolated turbines operating under the same wind inflow conditions. In order to cope with the effects of the aerodynamic interactions, one measure can be applied in WFs [6]. WTs on a WF are typically spaced farther apart in the direction parallel to the prevailing WD, known as downwind spacing, than in the perpendicular direction, known as crosswind spacing, as shown in Fig. 5.

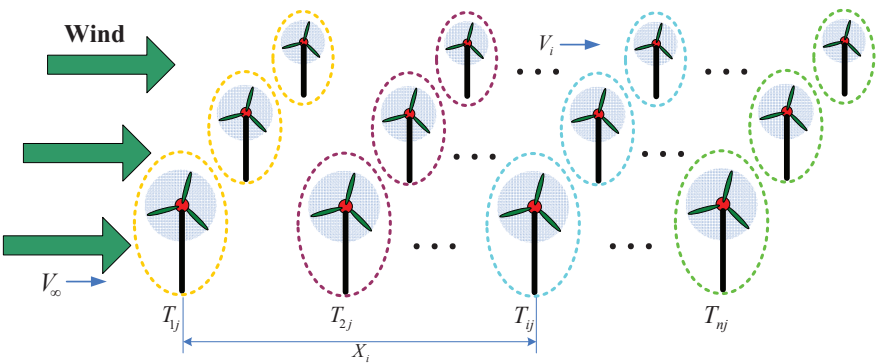

Fig. 5. The portrait of three-dimensional array of WTs in Luxembourg.

We can influence the power $P_{i}$ of turbine $i$ with the two control parameters $\lambda_{r i}$ and $\beta_{r i}$. Those are the reference points which the WT needs to track. For a WT operating in steady state at $\lambda_{i}=\lambda_{r i}$ and $\beta_{i}=\beta_{r i}$ (10) and (7) result in the same value. The total power $P_{\text {tot }}$ generated by a WF is:

$$
P_{\text {tot }}=\sum_{i \in N} P_{i}\left(\lambda_{r i}, \beta_{r i}\right)
$$

In the following section, we describe the model of the WF power generation process as shown in Fig. 5.

\section{An Unified COOPERATION WAKe Model (UCWM) FOR WF}

A unified cooperation model for WF wake is given in this section. At first, we introduce the Wake Interaction Model A (WIMA). The set of axial induction factors of each turbine is $a=\left\{a_{i} \mid i \in N\right\}$. Giving a single turbine $i$ with a rotor diameter $D_{i}$ and its radius $R$, with its rotor axis aligned with the WD. Suppose an incoming uniform wind field with a free-stream speed $V_{\infty}$. Let $(s, r)$ be a position of the turbine in WIMA, where $s$ is the distance to the rotor disk plane of the turbine, and $r$ is the distance to the centerline of the WT rotor axis (see Fig. 6) [5]. Then The WIMA estimates the wind speed in this point to be:

$$
V_{w, i}\left(s, r, a_{i}\right)=V_{\infty}\left[1-\delta V_{w, i}\left(s, r, a_{i}\right)\right] .
$$

where $\delta V_{w, i}\left(s, r, a_{i}\right)$ represents the fractional deficit of the velocity at the point $(s, r)$ downstream of turbine $i$ :

$$
\delta V_{w, i}\left(s, r, a_{i}\right)= \begin{cases}2 a_{i}\left(\frac{D_{i}}{D_{i}+2 k s}\right)^{2}, & \forall r \leq \frac{D_{i}+2 k s}{2}, \\ 0, & \forall r>\frac{D_{i}+2 k s}{2} .\end{cases}
$$

where $k$ represents a tunable wake expansion coefficient or a roughness coefficient. The $D_{w, i}$ is the diameter of the wake, which is assumed to have a circular cross-section. The diameter is assumed to expand proportional to the distance $s$ is: $D_{w, i}(x)=D_{i}+2 k s$.

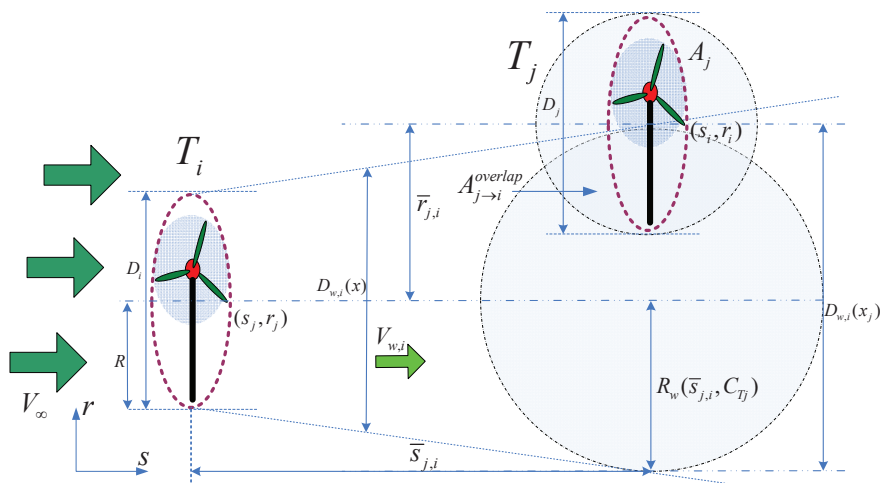

Fig. 6. The portraits of the interaction and coordination with the wake expansion parameters in UCWM.

The WIMA can be extended to include multiple turbines with interacting wakes. Then the effective wind speed $V_{i}$ for a turbine $i \in N$ is calculated by summing the velocity deficit created by the wakes of each upstream turbine:

$$
\begin{aligned}
V_{i}(a) & =V_{\infty}\left(1-\delta V_{i}(a)\right) \\
& =V_{\infty}\left(1-2 \sqrt{\sum_{j \in N ; x_{j}<x_{i}}\left(a_{j} b_{j i}\right)^{2}}\right) .
\end{aligned}
$$

where

$$
b_{j i}=\left(\frac{D_{j}}{D_{j}+2 k\left(x_{i}-x_{j}\right)}\right)^{2} \frac{A_{j \rightarrow i}^{\text {overlap }}}{A_{i}} .
$$

Then the aggregate velocity deficit seen by turbine $i$ is

$$
\delta V_{i}(a)=2 \sqrt{\sum_{j \in N ; x_{j}<x_{i}}\left[a_{j}\left(\frac{D_{j}}{D_{j}+2 k\left(x_{i}-x_{j}\right)}\right)^{2} \frac{A_{j \rightarrow i}^{\text {overlap }}}{A_{i}}\right]^{2}} .
$$

where $A_{i}$ is the area of the disk generated by the blades of turbine $i$ and $A_{j \rightarrow i}^{\text {overlap }}$ is the area of the overlap between the wake generated by turbine $j$ and the disk generated by the blades of turbine $i$. See Fig. 6 for an illustration with two WTs. 
The Wake Interaction Model B (WIMB) are a simplified characterisation of the wake resulting from a single wind turbine [7]. Here we use a general formulation of wake effect to describe the wind velocity profile $V\left(\bar{s}_{j}, \bar{r}_{j}, C_{T j}\right)$ caused by a single turbine $j \in N$ of the form:

$$
V\left(\bar{s}_{j}, \bar{r}_{j}, C_{T j}\right)=V_{\infty}\left[1-\delta V\left(\bar{s}_{j}, \bar{r}_{j}, C_{T j}\right)\right] .
$$

where $\delta V\left(\bar{s}_{j}, \bar{r}_{j}, C_{T j}\right)$ is the fractional velocity deficit at the relative coordinate $(\bar{s}, \bar{r})$ downstream of turbine $j$. Most wake models characterise the wake deficit caused by turbine $j$ as a function of the thrust coefficient $C_{T j}\left(\lambda_{j}, \beta_{j}\right)$ and downstream distance to the vertex in WD $\bar{s}_{j}=s-s_{j}$ and in orthogonal direction $\bar{r}_{j}=\left|r-r_{j}\right|$ as illustrated in Fig. 6. The thrust coefficient $C_{T j}$ is a function of the tip speed ratio $\lambda_{j}$ and pitch angle $\beta_{j}$ of turbine $j$. It describes the wind speed deficit as linear function of the thrust coefficient of the form:

$$
\delta V= \begin{cases}\frac{1}{2} C_{T j}\left(1+\frac{\bar{s}_{j}}{4 R}\right)^{-1}, & \text { if } \quad \bar{r}_{j} \leq \sqrt{4 R^{2}+\bar{s}_{j} R} \\ 0, & \text { if } \bar{r}_{j}>\sqrt{4 R^{2}+\bar{s}_{j} R}\end{cases}
$$

with the wake radius $R_{w}\left(\bar{s}_{j}, C_{T j}\right)=\sqrt{4 R^{2}+\bar{s}_{j} R}$.

Based on the WIMA and WIMB, we have the following UCWM:

$$
\begin{aligned}
& V_{i}\left(s, r, a_{i}, \bar{s}_{j}, \bar{r}_{j}, C_{T j}\right) \\
& =\lambda_{1} V_{w, i}\left(s, r, a_{i}\right)+\lambda_{2} V_{i}\left(\bar{s}_{j, i}, \bar{r}_{j . i}, C_{T j, i}\right) \\
& =\lambda_{1} V_{\infty}\left[1-\delta V_{w, i}\left(s, r, a_{i}\right)\right]+\lambda_{2} V_{\infty}\left[1-\delta V_{i}\left(\bar{s}_{j, i}, \bar{r}_{j, i}, C_{T j, i}\right)\right]
\end{aligned}
$$

here, $\lambda_{1}$ and $\lambda_{2}$ are coefficients, they satisfy with $0 \leq \lambda_{1}, \lambda_{2} \leq$ 1. Among the aboved equation, the corresponding variables $\delta V_{w, i}\left(s, r, a_{i}\right)$ and $\delta V_{i}$ are as follows:

$$
\delta V_{w, i}\left(s, r, a_{i}\right)= \begin{cases}2 a_{i}\left(\frac{D_{i}}{D_{i}+2 k s}\right)^{2}, & \forall r \leq \frac{D_{i}+2 k s}{2}, \\ 0, & \forall r>\frac{D_{i}+2 k s}{2} .\end{cases}
$$

and

$$
\delta V_{i}\left(\bar{s}_{j, i}, \bar{r}_{j, i}, C_{T j, i}\right)= \begin{cases}\frac{1}{2} C_{T j, i}\left(1+\frac{\bar{s}_{j, i}}{4 R}\right)^{-1}, \\ 0, & \text { if } \bar{r}_{j, i} \leq \sqrt{4 R^{2}+\bar{s}_{j, i} R} \\ & \text { if } \quad \bar{r}_{j, i}>\sqrt{4 R^{2}+\bar{s}_{j, i} R}\end{cases}
$$

with the wake radius $R_{w}\left(\bar{s}_{j, i}, C_{T j, i}\right)=\sqrt{4 R^{2}+\bar{s}_{j, i} R}$.

\section{Wind Rose AND Wind WEIBULl PROBABILITY DENSITY DISTRIBUTION}

In this section, we describe and summarize the wind rose and wind Weibull probability density distribution focus on WFs of Luxembourg. We get the actual and real-time data from this Website [13]. These wind resource data are collected and sorted out in the following Figs. The Fig. 7 and Fig. 8 shows rose portraits of average WD and WD (North=0) in Luxembourg from Jan. 01 to Dec. 31, 2014. The probabilities of most WD from southwest which are more than $3 \%$ and approximate approaches to $4 \%$. Whereas, a small amount of
WD from northeast which are more than 3\% and approximate approaches to $4 \%$. Therefore, WTs should face to southwest in WFs of Luxembourg.

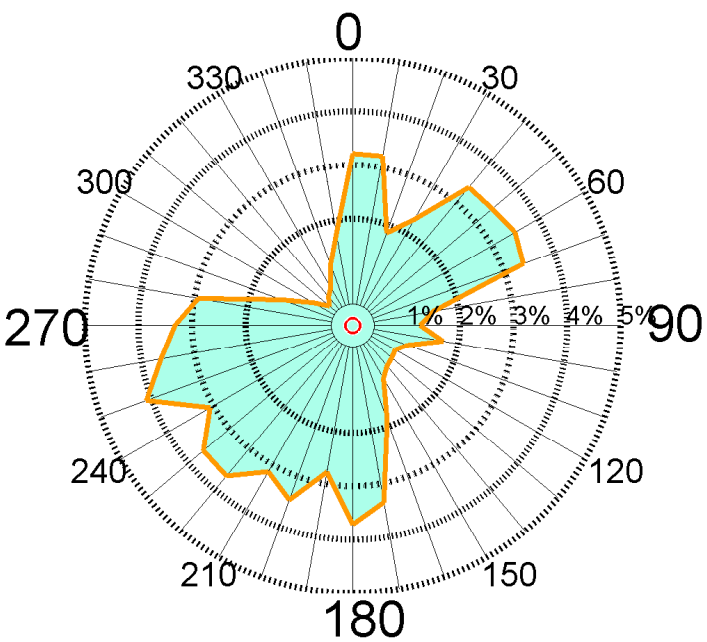

Fig. 7. Rose portrait of average WD(North=0) in Luxembourg from Jan. 01 to Dec. 31, 2014.

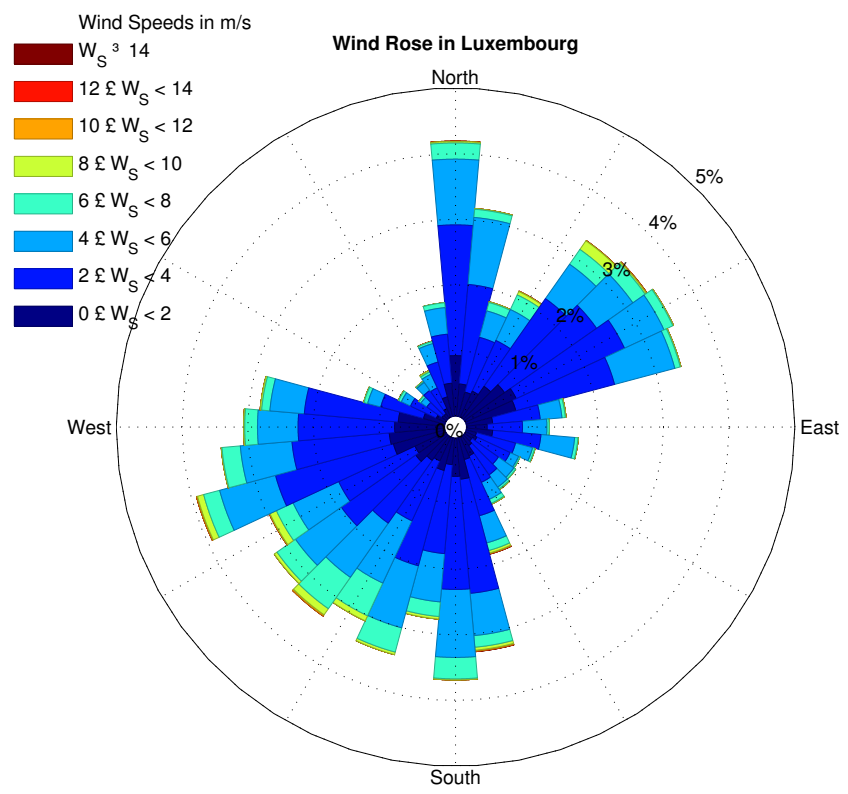

Fig. 8. Rose portrait of $\mathrm{WD}($ North $=0)$ in Luxembourg from Jan. 01 to Dec 31, 2014.

The wind rose shows the portraits of wind mean speed $(\mathrm{m} / \mathrm{s})$ in Luxembourg from Jan. 01 to Dec. 31, 2014 in Fig. 9. The most wind mean speed from southwest are more than $4 \mathrm{~m} / \mathrm{s}$. Whereas, a small amount of wind mean speed from northwest are approximate to $4 \mathrm{~m} / \mathrm{s}$. Therefore, wind turbines should face to southwest in WFs of Luxembourg.

We continue getting the actual and real-time data from this Website [13]. The wind speed in Luxembourg are collected from Jan. 01 to Dec. 31 in 2014 and their Mean Wind Speed 


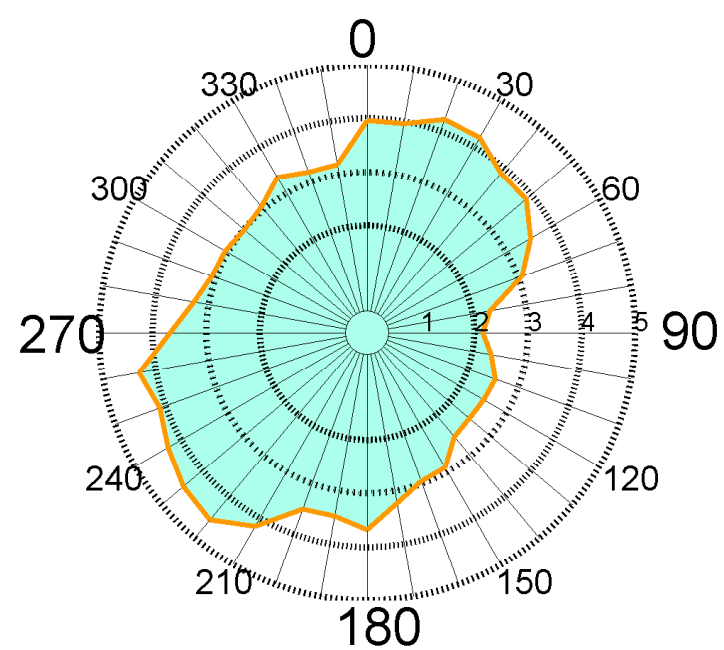

Fig. 9. Rose portrait of Wind Mean Speed(m/s) in Luxembourg from Jan. 01 to Dec. 31, 2014.

(MWS) is $3.2042 \mathrm{~m} / \mathrm{s}$. They are shown in the following Fig. 10.

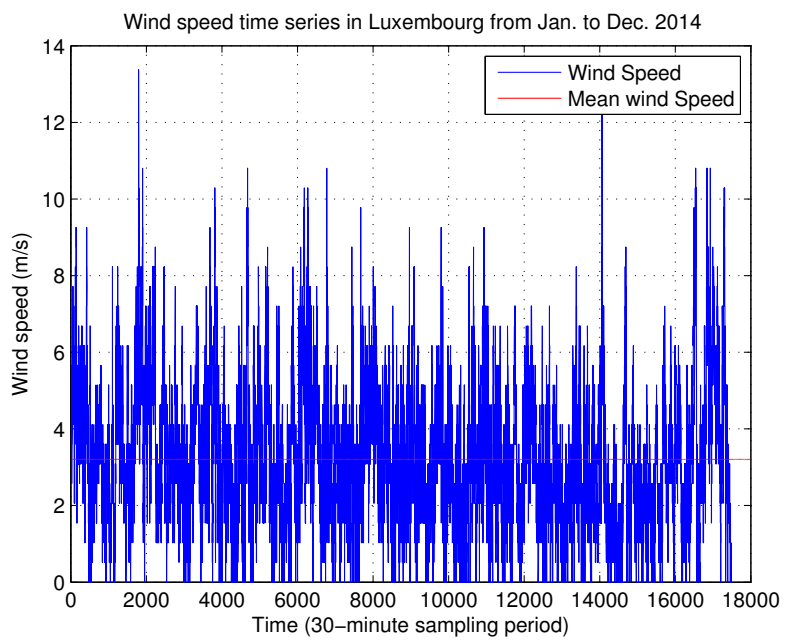

Fig. 10. The portraits of wind speed and mean wind speed $(=3.2042 \mathrm{~m} / \mathrm{s})$ in Luxembourg from Jan. 01 to Dec. 31, 2014.

Figs 11, 12, 13 and 14 show the the parameters and fitting curve of Weibull probability distribution for the wind speed in Luxembourg. According to wind tower, measuring data and draw wind speed histograms, Using Maximum likelihood estimation method, we estimate two parameters of the Weibull distribution, are that $c=3.4423$, and $k=1.9197$. Then we draw the Weibull probability density distribution curve in Figs 13 and 14. As seen in Figs 13 and 14, the probability distribution of wind speed can be more satisfied with the Weibull distribution.
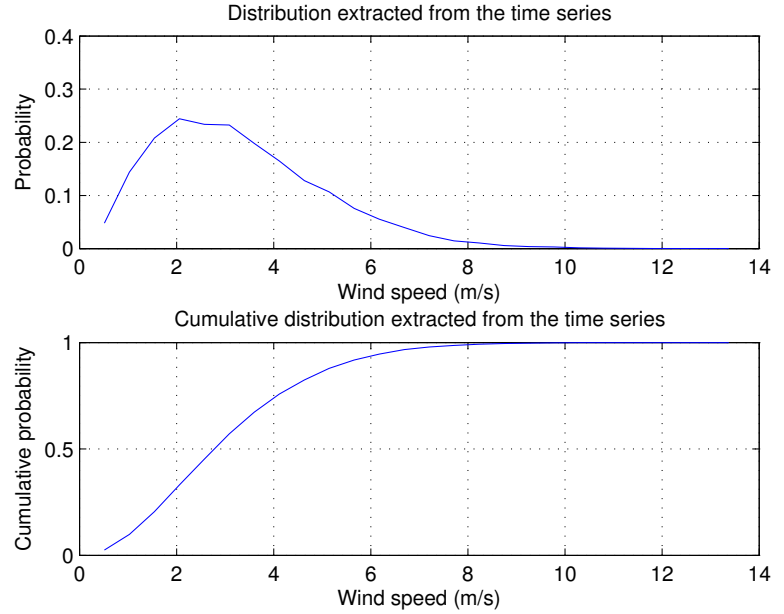

Fig. 11. The portraits of distribution and cumulative distribution extracted from the time series in Luxembourg from Jan. 01 to Dec. 31, 2014.

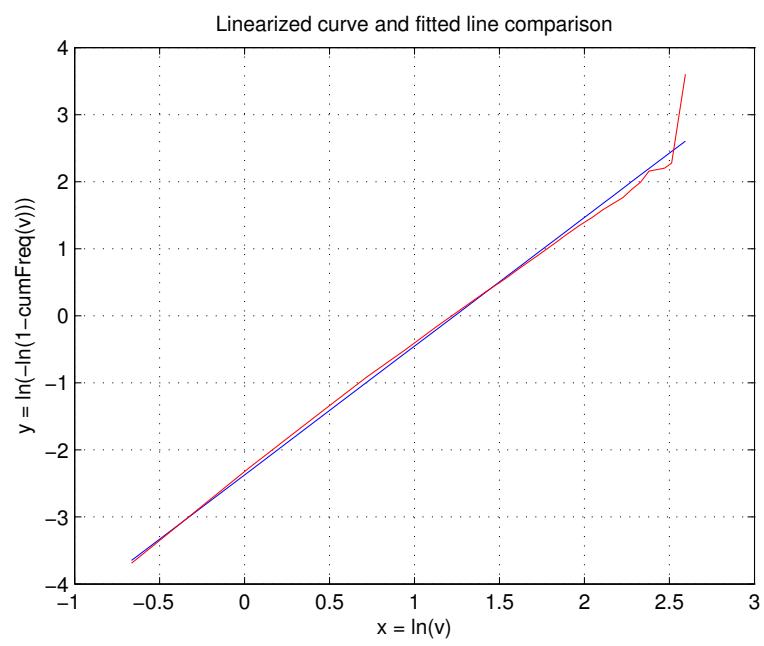

Fig. 12. The portraits of linearized curve and fitted line comparison in Luxembourg from Jan. 01 to Dec. 31, 2014.
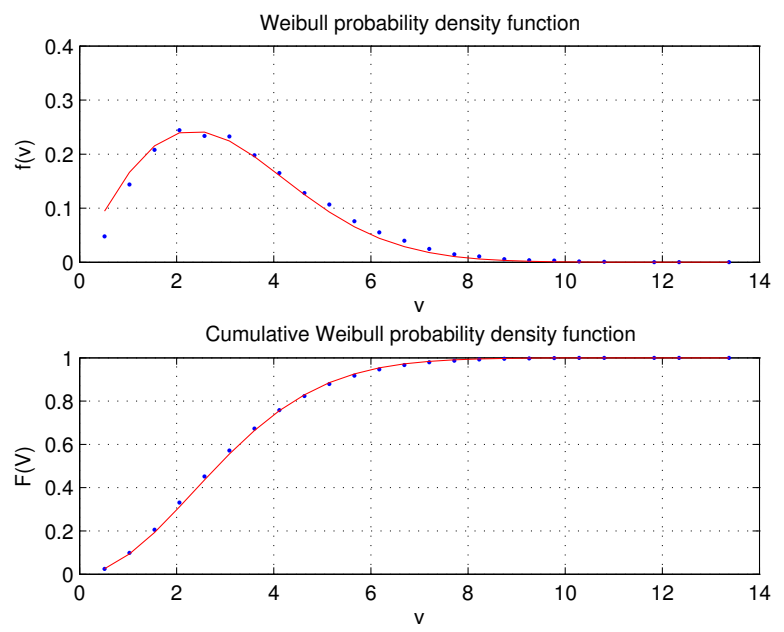

Fig. 13. The portraits of functions for Weibull probability density and Cumulative Weibull probability density in Luxembourg from Jan. 01 to Dec. 31, 2014. 


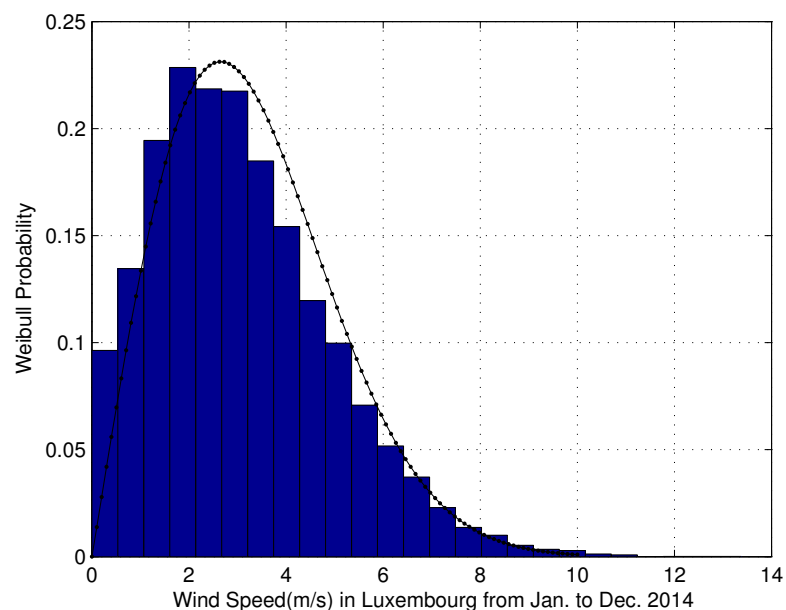

Fig. 14. The portraits of wind speed histogram in hub height and the fitted Weibull probability density distribution in Luxembourg from Jan. 01 to Dec. 31, 2014.

\section{CONCLUSIONS}

The paper investigates the WE systems in Luxembourg. We show the overview of the wind resources in all the WFs as well as present an Unified Cooperation Wake Model (UCWM) for WFs. Moreover, the statistical method is used to model the wind speed and WD distribution for WP assessment of WFs. Some simulation figures of the wind rose and Weibull distribution demonstrate the detailed description and assessment of WP. These assessments can effectively accelerate the process of WP development and utilization of WFs in Luxembourg. Next step, we will studied some new models which will be used to develop a distributed model predictive control approach for WFs. In addition, coordinated and optimization control of WFs will also be the aim of our research.

\section{ACKNOWLEDGMENT}

This work is supported by AFR and FNR programs.

\section{REFERENCES}

[1] C. Han, A. Huang, M. Baran, S. Bhattacharya, W. Litzenberger, L. Anderson, A. Johnson, and A. Edris, "Statcom impact study on the integration of a large wind farm into a weak loop power system," Energy Conversion, IEEE Transactions on, vol. 23, no. 1, pp. 226-233, March 2008.

[2] J. R. Marden, S. D. Ruben, and L. Y. Pao, "A model-free approach to wind farm control using game theoretic methods," IEEE Transactions on Control Systems Technology, vol. 21, no. 4, pp. 1207-1214, 2013.

[3] K. L. Sørensen, R. Galeazzi, P. F. Odgaard, H. Niemann, and N. K Poulsen, "Adaptive passivity based individual pitch control for wind turbines in the full load region," in Proceedings of the 2014 American Control Conference, Portland, Oregon, USA, June 4-6, 2014, pp. 554559.

[4] S. Kuenzel, L. Kunjumuhammed, B. Pal, and I. Erlich, "Impact of wakes on wind farm inertial response," Sustainable Energy, IEEE Transactions on, vol. 5, no. 1, pp. 237-245, Jan 2014.

[5] F. van Dam, P. Gebraad, and J.-W. van Wingerden, "A maximum power point tracking approach for wind farm control," Proceedings of The Science of Making Torque from Wind, 2012.

[6] L. Y. Pao and K. Johnson, "A tutorial on the dynamics and control of wind turbines and wind farms," in American Control Conference, 2009. ACC '09., June 2009, pp. 2076-2089.

[7] E. Bitar and P. Seiler, "Coordinated control of a wind turbine array for power maximization," in American Control Conference (ACC), 2013, June 2013, pp. 2898-2904.

[8] ENOVOS, "Enovos," http://www.enovos.eu.

[9] SEO, "Seo-energie," http://www.seo.lu.

[10] T. Wind Power, "The Wind Power," http://www.thewindpower.net/index.php.

[11] Z. Zhang and Y. Liang, "Constant output power control of variable trailing-edge flap wind power system based on feedback linearization," in Control Conference (CCC), 2014 33rd Chinese, July 2014, pp. 38053810.

[12] SimWindFarm, "Simwindfarm toolbox," http://www.ictaeolus.eu/SimWindFarm/.

[13] I. S. U. of Science and Technology, "The Iowa Environmental Mesonet (IEM)," http://mesonet.agron.iastate.edu/. 